\title{
Fetal Alcohol Spectrum Disorders must be recognized globally as a large public health problem
}

\section{Editorial}

Dear Readers,

We are pleased to start a new year by introducing the Second Special Issue on Fetal Alcohol Spectrum Disorders (FASD), which contains 11 original research papers.

According to the most recent Global Burden of Disease (GBD) and Injury study, alcohol was the fifth leading contributor to disability and mortality-3.9\% of global disability-adjusted life years (DALYs) and $5.2 \%$ of all global deaths were attributable to alcohol in 2010 (Lim et al., 2012). Furthermore, alcohol consumption often results in harm, not only to the drinker, but also to individuals associated with the drinker. A classic example is the harm caused by drinking during pregnancy.

The World Health Organization (WHO) global strategy to monitor and reduce the harmful use of alcohol, endorsed by the Sixty-Third World Health Assembly, highlights the importance of prevention and identification of the harmful use of alcohol among pregnant women and women of childbearing age, and treatment and care for individuals and families affected by FASD (WHO, 2010). However, although a substantial and growing body of scientific literature on FASD and related issues exists, current research data for many countries is scattered or meager. Consequently, in most countries FASD is not well recognized by health professionals, and prevention, treatment and care interventions are not being implemented or addressed at the policy and program levels. One of the main reasons for this is that, despite over 40 years' worth of evidence, FASD was not officially recognized as a medical diagnosis and was not included in either the Diagnostic and Statistical Manual of Mental Disorders (DSM) or the International Classification of Diseases (ICD; with the exception of Fetal Alcohol Syndrome).

Only very recently, in mid-2013, after a long battle, "Neurodevelopmental Disorder associated with Prenatal Alcohol Exposure [ND-PAE]" was included in the Appendix of the fifth edition of the DSM as a condition warranting future research before it can be considered for clinical use and inclusion in the DSM as a formal disorder. This is a step in the right direction, albeit a small one. The formal recognition of FASD will necessitate its inclusion in medical college curriculums and the initiation of mandatory training of health care providers, psychiatrists, psychologists, social workers, individuals working within the justice system, and other professionals, all of which will improve the diagnosis and monitoring of FASD. To include the entities that fall within the FASD spectrum in the DSM and the ICD would provide the impetus for further research, the development of new and more effective treatment methods, and an increase in the number of diagnostic clinics or the capacity of existing ones.

Despite the preventable nature of this group of disorders, it appears that the prevalence of FASD may increase around the globe in the coming years. This is due mainly two factors: first, the rates of alcohol use, binge drinking and drinking during pregnancy are increasing among young women in a number of countries (Lim et al 2012; Thomas, 2012; WHO, 2011), and second, the majority of pregnancies in developing and developed countries are unplanned (Alvanzo \& Svikis, 2008; Balachova et al., 2012; Hartley et al., 2011).

The harmful effects of alcohol on a fetus, representing many cases of preventable disability, should be recognized globally as a large public health problem. The present collection of research studies, briefly described below, clearly demonstrates the need for such recognition.

Balachova and colleagues present results from a survey conducted among 648 pregnant and non-pregnant women of childbearing age in two regions of Russia on the perceived determinants of alcohol consumption during pregnancy, and the women's trust in and receptivity to various sources of information. The findings from this study will provide decision makers and policy developers with important information about how prevention initiatives can be most effectively be implemented in Russia - a country with some of the highest rates of alcohol consumption in the world.

Callinan and Ferris, using data from the National Drug Strategy Household Survey in Australia over a 10-year period, explored the relationship between maternal age, the year of pregnancy, the year of birth, and alcohol use during pregnancy. The analysis revealed that the year of pregnancy was the strongest predictor of self-reported alcohol consumption during pregnancy. The proportion of 
women reporting alcohol use during pregnancy dropped from $44 \%$ in 2001 to $25 \%$ in 2007 , with $20 \%$ drinking after knowledge of pregnancy in 2010. This finding of a steady decrease in alcohol consumption during pregnancy in Australia in the past decade is very promising.

Using a mixed-method study design, Clark and colleagues evaluated the effectiveness of a professional development program for elementary school teachers supporting students with FASD in British Columbia, Canada. The findings from this exploratory study provide preliminary evidence to support the program's positive effects on the academic achievement and classroom behavior of the students with FASD, and its contribution to the development of teaching practice to better accommodate students' neurological deficits. However, further evaluation is needed to overcome the limitations of this study.

Gindis used quantitative and qualitative clinical data, collected at non-governmental clinics in New York and Arizona, U.S.A., on medical, neuropsychological, and educational assessments of post-institutionalized children adopted from Europe with and without FASD. The findings from this study have important implications for adoptive parents and educational systems.

Grant and colleagues examined substance abuse inpatient and outpatient treatment participation and completion rates in different treatment settings in the state of Washington, U.S.A., comparing rates for women with diagnosed and suspected FASD to those of women who were not exposed to alcohol in utero. The study revealed that women with diagnosed or suspected FASD were less likely to participate and complete inpatient and outpatient treatment than were those without prenatal alcohol exposure. The findings of this study are important for effective program implementation; they can be used to make program adaptations that target the needs of those with FASD who are abusing drugs and/or alcohol and can consequently prevent future "second generation" cases of FASD.

The study by Hicks and colleagues used data obtained from 2,000 pregnant women attended by family physicians at low-risk maternity clinics in Alberta, Canada. The results support the use of the T-ACE screening questionnaire in routine care to identify women who are at risk not only for alcohol misuse, but also for mental health problems, poor social support, and other detrimental lifestyle factors. Once identified, these pregnant women with complex needs may benefit from additional prenatal support.

In an attempt to support program transferability, implemented in a small, rural community in British Columbia, Canada, Hubberstey and colleagues present preliminary evaluation findings for a Youth Outreach Program designed for at-risk Aboriginal youth with suspected FASD. Given the promising results described, this program may be recommended to marginalized and disadvantaged youth with prenatal alcohol exposure/FASD who have limited family and community support.
Job and colleagues from Alberta, Canada discuss the findings of an innovative methodological approach as a means of accessing the FASD prevention specialists' programmatic experiences. This novel practice, which utilizes visual methods, has the potential to inform FASD policy, strategic planning, and programming.

Rutman and colleagues present three frameworks, designed to evaluate 1) FASD prevention programs, 2) FASD support programs, and 3) FASD programs in Aboriginal communities. This Canadian project produces visual maps that have been found to promote both individual and collective efforts and have a wide range of applicability.

In an attempt to determine the effectiveness of alcohol warning labels in preventing alcohol use during pregnancy, Thomas and colleagues performed a review of published academic and web-based literature. The authors provide important findings, which can be used in developing welldesigned, effective FASD prevention strategies.

Lastly, Williams and colleagues investigated the prevalence of smoking during pregnancy, and its relationship with prenatal alcohol consumption, among more than 3,000 pregnant women in the Republic of the Congo. The finding that cigarette smoking at any level is associated with an almost five-fold increase in risk for alcohol use during pregnancy has the potential to inform targeted prevention programs and initiatives.

We truly hope that the special issues on FASD will increase awareness of the detrimental effects of alcohol consumption during pregnancy, inform policies and program development to benefit those living with FASD, and facilitate prevention activities on drinking among pregnant women and women of childbearing age, to prevent further alcohol-affected births around the world.

We also hope that the research presented here will further inspire international collaborations of scientists and health care providers working within the field of FASD and maternal substance use.

\section{References}

Alvanzo, A. A. H., \& Svikis, D. S. (2008). History of physical abuse and periconceptional drinking in pregnant women. Substance Use and Misuse, 43, 1098-1109.

Balachova, T., Bonner, B. L., Chaffin, M., Bard, D., Isurina, G., Tsvetkova, L., \& Volkova, E. (2012). Women's alcohol consumption and the risk for alcohol-exposed pregnancies in Russia. Addiction, 107, 109-117.

Hartley, M., Tomlinson, M., Greco, E., Comulada, W. S., Stewart, J., le Roux, I., . . . Rotheram-Borus, M. J. (2011). Depressed mood in pregnancy: Prevalence and correlates in two Cape Town peri-urban settlements. Reproductive Health, 8, 9.

Lim, S. S., Vos, T., Flaxman, A. D., Danaei, G., Shibuya, K., Adair-Rohani, H., . . . Ezzati, M. (2012). A 
comparative risk assessment of burden of disease and injury attributable to 67 risk factors and risk factor clusters in 21 regions, 1990-2010: A systematic analysis for the Global Burden of Disease Study 2010. Lancet, 380(9859), 2224-2260. (Errata published 2013, in Lancet 381(9874), 1276; Lancet 381(9867), 628.)

Thomas, G. (2012). Levels and patterns of alcohol use in Canada. Alcohol Price Policy Series. Report 1. Ottawa, ON: Canadian Centre on Substance Abuse. Retrieved from http://www.ccsa.ca/2012\%20CCSA\% 20Documents/CCSA-Patterns-Alcohol-Use-PolicyCanada-2012-en.pdf.

World Health Organization (WHO). (2010). Sixty-Third World Health Assembly. Geneva, Switzerland: WHO.

World Health Organization (WHO). (2011). Global Status Report on Alcohol and Health. WHO: Geneva, Switzerland: WHO. Retrieved from http://www.who.int/substance_abuse/publications/glob al_alcohol_report/profiles/rus.pdf

Svetlana (Lana) Popova, M.D., Ph.D., M.P.H.

Senior Scientist, Social and Epidemiological Research,

Centre for Addiction and Mental Health

Assistant Professor, Epidemiology Division,

Dalla Lana School of Public Health, University of Toronto

Assistant Professor, Factor-Inwentash Faculty of Social

Work, University of Toronto

Graduate Faculty Associate Member,

Institute of Medical Science, University of Toronto

Toronto, Canada

Christina Chambers, Ph.D., M.P.H.

Professor

Departments of Pediatrics and Family and Preventive Medicine

and Skaggs School of Pharmacy and Pharmaceutical Sciences

University of California San Diego, La Jolla, CA

Director of Research, Rady Children's Hospital, San Diego, CA 